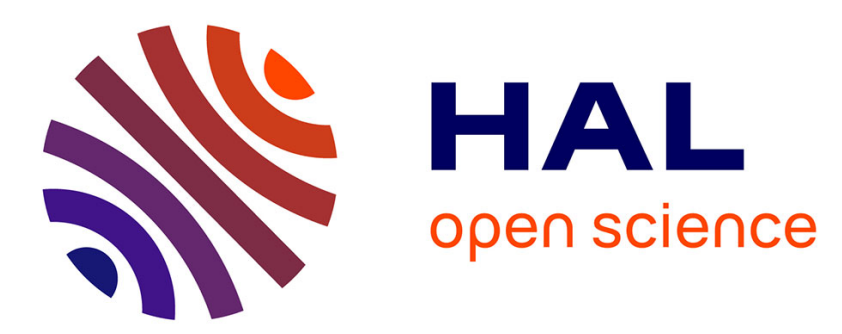

\title{
Structural transitions in ultrathin binary mixture films: an interplay between finite size and surface effects
}

\author{
Jean-Pierre Desideri, Didier Sornette
}

\section{To cite this version:}

Jean-Pierre Desideri, Didier Sornette. Structural transitions in ultrathin binary mixture films: an interplay between finite size and surface effects. Journal de Physique, 1988, 49 (8), pp.1411-1423. 10.1051/jphys:019880049080141100 . jpa-00210821

\section{HAL Id: jpa-00210821 \\ https://hal.science/jpa-00210821}

Submitted on 1 Jan 1988

HAL is a multi-disciplinary open access archive for the deposit and dissemination of scientific research documents, whether they are published or not. The documents may come from teaching and research institutions in France or abroad, or from public or private research centers.
L'archive ouverte pluridisciplinaire HAL, est destinée au dépôt et à la diffusion de documents scientifiques de niveau recherche, publiés ou non, émanant des établissements d'enseignement et de recherche français ou étrangers, des laboratoires publics ou privés. 
Classification

Physics Abstracts

$67.60 \mathrm{~F}-68.15-67.70$

\title{
Structural transitions in ultrathin binary mixture films : an interplay between finite size and surface effects
}

\author{
Jean-Pierre Desideri and Didier Sornette \\ Laboratoire de Physique de la Matière condensée, CNRS UA 190, Faculté des Sciences, Parc Valrose, \\ 06034 Nice Cedex, France
}

(Reçu le 6 janvier 1988, accepté le 29 mars 1988)

\begin{abstract}
Résumé. - Nous proposons un modèle pour les transitions homogène-stratifié observées dans les films de mélange $\mathrm{d}^{3}{ }^{3} \mathrm{He}-{ }^{4} \mathrm{He}$ utilisant la technique du troisième son. On présente un développement de type LandauGinzburg contenant la contribution des interactions atomes-atomes et celle de l'ordre superfluide ainsi que des termes de surface et d'interface avec en plus la condition de conservation des espèces ${ }^{3} \mathrm{He}$ et ${ }^{4} \mathrm{He}$. Les résultats obtenus suggèrent des images précises de la structure des films déduites de l'analyse des expériences et soulignent le rôle de la compétition entre les termes de volume, de surface et d'interface dans ce régime d'épaisseurs comprises entre 3 et 20 couches atomiques. Le comportement de ces films apparaît ainsi intermédiaire entre bi- et tri-dimensionnel et est caractéristique d'effets de taille finie.
\end{abstract}

\begin{abstract}
We propose a simple model for the homogeneous-stratified transitions observed in ${ }^{3} \mathrm{He}-{ }^{4} \mathrm{He}$ binary mixture films using the third sound technique. A continuous Landau-Ginzburg model with a bulk term containing both atom-atom and superfluid contributions plus surface and interface terms with in addition the constraint of conservation of helium 3 and helium 4 is presented and its predictions compared to experiments. Our results suggest precise pictures for the different film structures deduced from the analysis of experiments and stress the intricate interplay between bulk, surface and interface energies as occurs in this range of thicknesses ( 3 atomic layers $\leqslant d \leqslant 20$ atomic layers). The behaviour of these films appears to be intermediate between two and three dimensional and points at the importance of finite size effects.
\end{abstract}

\section{Introduction.}

${ }^{3} \mathrm{He}-{ }^{4} \mathrm{He}$ mixtures constitute ideal paradigms for studying the challenging physics of binary systems in the presence of surfaces (leading to wetting [1] and surface transitions [2]...), in restricted geometries (leading to capillary condensation [3]...) and in low dimensions. The phase diagram of bulk ${ }^{3} \mathrm{He}-{ }^{4} \mathrm{He}$ mixtures is well documented as is the study of various surface transitions (wetting and superficial superfluid transitions [4]...) occurring when the bulk phase is in contact with a wall. Also, quasi-two dimensional films a few atomic layers thick exhibit a vortex-mediated superfluid transition [5] in agreement with the Kosterlitz-Thouless scenario [6].

On the other hand, much less is known about the cross-over between two- to three-dimensional behaviour with respect to the superfluid order, to the ${ }^{3} \mathrm{He}$ and ${ }^{4} \mathrm{He}$ concentration order and in the presence of a superfluid-concentration coupling. ${ }^{3} \mathrm{He}-{ }^{4} \mathrm{He}$ films are probably the best systems for studying these problems and a wealth of experiments have been concerned with them. The structure of thin films as a function of thickness, concentration and temperature is not yet known precisely : in particular the existence and the nature of phase separations in mixture films are still a debated question. From the complexity and richness of experimental results, we can distinguish between several regimes depending on the values of the ${ }^{3} \mathrm{He}$ and ${ }^{4} \mathrm{He}$ thicknesses $d_{3}$ and $d_{4}$ and the temperature :

1) for very dilute mixture films $d_{3}<0.2$ a.l. (a.1. = atomic layers), heat capacity measurements [7] suggest the existence of a lateral phase separation occurring at the surface of the film. The states of the ${ }^{3} \mathrm{He}$ atoms should be controlled by the discrete Andreev levels [8] which merge into a continuum as $d_{4}$ goes to infinity. Also, third sound velocity measurements can be affected by the ${ }^{3} \mathrm{He}$ impurities which could be trapped in Kosterlitz-Thouless vortices [9] ; 
2) for very small $d_{4}\left(d_{4} \leqslant 2-2.5\right.$ a.1. $)$, a dead solid helium layer $(\approx 1 \mathrm{a} .1$. thick $)$ in contact with the substrate, which is created by the strong Van der Waals attraction, contains a significant proportion of the total ${ }^{4} \mathrm{He}$ of the film : new mechanisms such as ${ }^{4} \mathrm{He} \leftrightarrow{ }^{3} \mathrm{He}$ exchanges in the solid layer could be at work and explain the survival of superfluidity at such low thicknesses [10] ;

3) for mixture films with $d_{3} \gg 1$ and $d_{4}$ still of the order of a few atomic layers such that superfluidity cannot exist if the film is in a homogeneous state, a layered phase separation has been observed [5]. This is reminiscent of the superficial superfluid transition [4] occurring when a bulk ${ }^{4} \mathrm{He}-{ }^{3} \mathrm{He}$ mixture just oustide the region of phase separation is in contact with a wall (see below);

4) in the intermediate regime $\left(d_{3} \approx 1-5\right.$ a.1., $d_{4} \approx$ 2-6 a.1.), a very rich phenomenology has been reported [11-14]. For ${ }^{4} \mathrm{He}$ coverage equal to 5.7 a.1., mixture films exhibit a nearly complete layered phase separation [13]. More precise studies [12] have explored the film structures as $d_{3}$ and $d_{4}$ are varied : a continuous but rather steep crossover from a homogeneous to an apparently stratified state has been observed as $d_{4}$ increases from 3 to 6 a.l. (see Fig. 4). Note however that these third sound experiments were analysed assuming that the superfluid and the normal phases are respectively pure ${ }^{4} \mathrm{He}$ and pure ${ }^{3} \mathrm{He}$ in the layered state, a hypothesis which is not sustained by measurements of the direct superfluid mass $\sigma_{\mathrm{s}}$ per unit area obtained by torsional oscillator techniques [5]. In particular, the strong temperature dependence of $\sigma_{\mathrm{s}}$ led McQueeney et al. to the conclusion that the two phases in the layered film structure are not just pure ${ }^{4} \mathrm{He}$ and pure ${ }^{3} \mathrm{He}$.

In this work, we study the fourth regime theoretically and argue that the corresponding experimental results can be rationalized within a simple model of the film structure embodying the different competing contributions of the bulk (atom-atom plus superfluid order), surface and interface energies. We suggest that regimes (3) and (4) belong to the general class of surface or wetting regimes in the presence of finite size effects [15] and present a model for the different homogeneous-stratified transitions observed in ${ }^{3} \mathrm{He}-{ }^{4} \mathrm{He}$ binary mixture films. In the spirit of surface or wetting phase transitions of binary mixtures in the presence of a wall, we use a continuous LandauGinzburg expansion capturing the contributions of the bulk free energy of the binary mixture and of both the surface and interface energies with the constraint of conservation of ${ }^{3} \mathrm{He}$ and ${ }^{4} \mathrm{He}$ in the film. Continuous ${ }^{3} \mathrm{He}$ concentration profiles as a function of the distance from the substrate are obtained which exemplify the complex ${ }^{4} \mathrm{He}-{ }^{3} \mathrm{He}$ mixed structure in the films. By comparison with third sound experiments, our results suggest that the structural transitions reflect the intricate interplay between bulk, surface and interface energies that occurs in this range of thicknesses $(3 \leqslant d \leqslant 20)$ intermediate between true two and three dimensional behaviour. In other words, the behaviour of the mixture film structure belongs to the class of finite size effects and, in the corresponding thickness range, exhibits non-universal features.

In paragraph 2, we develop the Landau-Ginzburg model, expose in paragraph 3 the results of the numerical solutions of the mean field equations and compare them to experiments. Paragraph 4 concludes by pointing out the limitations of the present approach and discusses its connection with a previous proposed model presented in [12]. The appendix gives a new derivation of the third sound velocity in inhomogeneous superfluid films.

\section{The Landau-Ginzburg model.}

2.1 INGREDIENTS. - Our main idea is that, sufficiently far from the tricritical point, a mixture film is essentially a two-phase system in a restricted (ultrathin slab) geometry [16]. This implies that the film structure should result from the competition between 1) a bulk free energy describing the bulk ${ }^{3} \mathrm{He}-{ }^{4} \mathrm{He}$ interactions, entropy and superfluid order leading to bulk phase separation, 2) the surface (substrate and liquid-gas) energy contributions and 3) the energy cost for creating spatial variations of the ${ }^{4} \mathrm{He}-{ }^{3} \mathrm{He}$ concentrations and superfluid order parameter along the direction perpendicular to the film. These are the ingredients of the well-studied wetting [1] or surface transitions [2] in usual binary mixtures, of the proximity effects in supraconductors [17] and of the healing length origin [18] for a superfluid in the presence of an interface. In films, however, the problem is more involved and exhibits specific features due to the extreme thinness of the film (leading to true two-dimensional or to finite size corrections to three dimensional behaviour, depending on the thickness of the film), the asymmetry between the two surfaces. (substrate-liquid and liquid-gaz interfaces), the coupling between the concentration and the superfluid order parameters and the resulting bulk tricritical behaviour.

2.2 ORDER PARAMETERS. - Let us denote $X_{3}$ the mean ${ }^{3} \mathrm{He}$ film concentration, $X(z)$ the ${ }^{3} \mathrm{He}$ concentration at distance $z$ from the substrate $(0 \leqslant z \leqslant d$, where $d$ is the total film thickness counted in atomic layers (a.1.)) and $\Psi=A \mathrm{e}^{i \varphi}$ the superfluid order parameter at $z$. We define the coarse-grained space dependent concentration order parameter $\Phi(z)$ by

$$
\Phi(z)=X(z)-X_{3}
$$

$\Phi=0$ corresponds to a strictly homogeneous film. 
The superfluid order is less easy to take into account. For two-dimensional films, the amplitude $A$ whose square gives the bare superfluid density is usually taken as constant (except at the core of the vortices) and the phase $\varphi$ is related to the superfluid velocity : its non-single-valuedness corresponds to the presence of topological defects, the vortices. At small scales (typically of the order of the bulk superfluid correlation length $\xi_{\mathrm{s}}$ ), $A$ may vary notably and for example it vanishes at the core of the vortices over the scale $\xi_{\mathrm{s}}$. This small-scale physics can be described within a Ginzburg-Pitaevsky expansion in terms of $A$, from which the value of the energy needed to create a neutral vortex pair can be estimated [19]. However, at large scales, the intricate renormalized interactions between vortices are at the basis of the defects-mediated Kosterlitz-Thouless 2D superfluid transition. Within this scenario, it is an open problem to describe the influence of the film structure on the superfluid order. Since we are concerned with thin films of thicknesses 3 a.l. $\leqslant$ $d \leqslant 20$ a.l. typically, one may decouple the small scale physics in the $z$-direction from the large scale in plane two-dimensional physics. This is an approximation but it is reasonable and leads to a simple treatment as we see below. We therefore follow Ginzburg and Pitaevskii [18] and use an expansion of the wave function $\Psi$ of the superfluid helium. This treatment does not claim to be describing the macroscopic superfluid state of the film but aims at estimating the superfluid contribution to the film free energy which conditions its structure.

2.3 The Hamiltonian. - The Landau-Ginzburg free energy reads :

$$
F\{\Phi(z), \Psi(z)\}=\int \mathrm{d}^{2} r \int_{0}^{d} \mathrm{~d} z f(\Phi(z), \Psi(z))
$$

with

$$
\begin{aligned}
f(\Phi(z) & , \Psi(z))= \\
= & {\left[\left\{G(\Phi, \Psi)+(1 / 2) \xi_{\mathrm{c}}^{2}(\mathrm{~d} \Phi / \mathrm{d} z)^{2}\right\}\right.} \\
& +G_{\mathrm{s}}^{\prime}(\Phi) \delta(z)+G_{\mathrm{s}}^{\prime \prime}(\Phi) \delta(z-d) \\
& \left.+\left(h^{2} / 8 \pi^{2} m^{* 2}\right)|\nabla \Psi|^{2}+\gamma \Phi \cdot|\Psi|^{2}\right]
\end{aligned}
$$

$G(\Phi, \Psi)$ is the bulk free energy density. The term $(1 / 2) \xi_{\mathrm{c}}^{2}(\mathrm{~d} \Phi / \mathrm{d} z)^{2}$ accounts for the energy cost of non-uniform ${ }^{3} \mathrm{He}$ concentrations stemming from a disruption of the local order of the helium fluid. $\xi_{\mathrm{c}}$ sets the typical scale for the bulk concentration correlation length (with $G(\Phi, \Psi)=\alpha(T) \Phi^{2}$, in mean field the bulk concentration correlation length scales as $\xi_{c}[\alpha(T)]^{-1 / 2}$ ). It is at the origin of the existence of interfaces between two phases whose surface tension and width are completely determined from $\xi_{\mathrm{c}}$. The terms $G_{\mathrm{s}}(\Phi)$ take into account the coupling between the surfaces and the concentration order parameter defined in equation (1). The term proportional to $|\nabla \Psi|^{2}$ is the usual expansion of the thermodynamic superfluid potential. It is at the origin of the proximity effects in superconductors [17] and of the healing length origin [18] for superfluids in contact with an interface and it allows one to compute the core energy of vortices. The third term $\gamma \Phi,|\Psi|^{2}$ describes the coupling between concentration and superfluid orders (see [20]).

\subsection{REDUCTION TO A SINGLE ORDER PARAMETER.}

The film structure should be obtained from the full calculation of the free energy which depends on the two coupled fields $\Phi$ and $\Psi$ in the presence of suitable boundary conditions for the superfluid order parameter. Due to the Kosterlitz-Thouless nature of the superfluid order and because of the existence of an additional constraint of conservation of helium (see below), this program cannot be pursued without drastic simplifications.

It is well known that superfluidity couples strongly with concentration ordering, resulting for example in a critical temperature for the bulk ${ }^{4} \mathrm{He}-{ }^{3} \mathrm{He}$ mixture at $T_{\mathrm{c}}=0.87 \mathrm{~K}$ much higher than the value in the absence of coupling $T_{\mathrm{c}} \approx 0.1 \mathrm{~K}$ estimated from the typical He-He interaction strength [21]. In the limit of strong coupling between superfluidity and ${ }^{4} \mathrm{He}$ concentration, we have

$$
1-\Phi=|\Psi|^{2}
$$

which allows one to replace the two field problem by a single order parameter $\Phi$. Equation (3) is not strictly correct everywhere in the film due to the different influences of a substrate on $\Phi$ and $\Psi$ (see [20]) but is a reasonable starting point. By its very nature, equation (3) is unable to describe the layered asymptotic model where a pure ${ }^{3} \mathrm{He}$ layer lies on top of a pure ${ }^{4} \mathrm{He}$ layer in contact with the substrate. This is the main limitation of our present approach which could be cured by considering $\Phi$ and $\Psi$ as two independent order parameters. In the following, we assume equation (3) to be valid ; this therefore limits our analysis to an intermediate concentration range (see below).

Far away from the tricritical point where the superfluid correlation length $\xi_{\mathrm{s}}$ is small, expression (3) can also be viewed as a kind of adiabatic approximation where the superfluid order parameter follows the concentration order parameter adiabatically. This amounts to having $\partial^{2} \Psi / \partial z^{2}=0$ in the Euler-Lagrange equation which yields $\Psi(z)=$ $\Psi(\Phi(z))$. The problem is then posed uniquely in terms of $\Phi$. This simplification should retain the main qualitative features of the problem.

\subsection{EXPLICIT EXPRESSION OF THE DIFFERENT CON-} TRIBUTIONS. - We choose $G\left(\Phi,|\Psi|^{2}\right)=G(\Phi)$ as the bulk energy density which describes the bulk 
phase diagram of ${ }^{4} \mathrm{He}-{ }^{3} \mathrm{He}$ mixtures : at temperatures lower than $2.17 \mathrm{~K}$ and for ${ }^{3} \mathrm{He}$ molar concentrations inside the bulk coexistence diagram, bulk mixtures split into two phases, one which is superfluid with a ${ }^{3} \mathrm{He}$ concentration $X_{\mathrm{S}}(T)$, and the other, a classical liquid with the ${ }^{3} \mathrm{He}$ concentration $X_{\mathrm{N}}(T)$. Setting

$$
\Phi_{\mathrm{S}}=X_{\mathrm{S}}(T)-X_{3}
$$

and

$$
\Phi_{\mathrm{N}}=X_{\mathrm{N}}(T)-X_{3}
$$

scaling arguments [22] suggest the following expression for $G(\Phi)$, which must display a minimum for $\Phi=\Phi_{\mathrm{S}}$ and $\Phi=\Phi_{\mathrm{N}}$ :

$$
G(\Phi)=a\left(\Phi-\Phi_{\mathrm{S}}\right)^{2}\left(\Phi-\Phi_{\mathrm{N}}\right)^{2}
$$

where $a=a(T)$ is a slowly varying function of the temperature. We have verified that our main results are not sensitive to the specific form of (5) provided it describes the essential features of the bulk ${ }^{4} \mathrm{He}-{ }^{3} \mathrm{He}$ phase diagram (i.e. the phase separation).

For the surface contribution $G_{\mathrm{s}}(\Phi)$, we choose

$$
\begin{aligned}
& G_{\mathrm{s}}^{\prime}(\Phi(0))=h_{\mathrm{s}} \Phi(0) \\
& G_{\mathrm{s}}^{\prime \prime}(\Phi(d))=0 .
\end{aligned}
$$

Expression (6) embodies the influence of the Van der Waals interactions between the liquid and the substrate with preferential attraction of ${ }^{4} \mathrm{He}$ onto the substrate due to its smaller effective size created by zero point motion [23]. With $h_{\mathrm{s}}>0$, this energy is lowered when $\Phi(0)<0$, i.e. when the local ${ }^{4} \mathrm{He}$ concentration becomes larger on the substrate than in the bulk.

The scale of the surface field $h_{\mathrm{s}}$ on the substrate at $z=0$ is roughly given by $\gamma_{33}^{\text {ls }}-\gamma_{44}^{\text {ls }}$ where $\gamma_{33}^{\text {ls }}$ is the liquid-solid surface tension of bulk ${ }^{3} \mathrm{He}$. For the liquid gas interface, we would have $\mathrm{h}_{\mathrm{s}}^{\mathrm{lg}} \approx \gamma_{33}^{\mathrm{lg}}-$ $\gamma_{44}^{\text {lg }}$ which is much smaller than $h_{\mathrm{s}}$ by the ratio of the typical strength of the He-He Van der Waals interaction $(\approx 3 \mathrm{~K})$ to the He-substrate Van der Waals interaction $(\approx 30-50 \mathrm{~K})$. This justifies our choice of a liquid-gas surface field $h_{\mathrm{s}}^{\mathrm{lg}} \approx 0$ and leads to equation (7). We have verified that the results are not qualitatively changed by adding a surface field at $z=d$ (which favors ${ }^{3} \mathrm{He}$ due to its larger atomic size leading to a smaller liquid-gas surface tension). Note that the most general expression for $G_{\mathrm{s}}(\Phi)$ should contain an additional term of the form $(1 / 2) \Phi_{s}^{2}$ which describes the disordering effect of the presence of a wall bound to suppress all $\mathrm{He}-\mathrm{He}$ interactions between the film atoms and a hypothetical half infinite space filled with helium taking the place of the substrate. For this preliminary study, we drop this term for the sake of simplicity. Lipowsky and Kroll [24] have demonstrated that the two formulations of surface phase transitions in terms of either the surface film (6) or the disordering term (1/2) $\Phi_{\mathrm{s}}^{2}$ are equivalent. $h_{\mathrm{s}}$ is taken equal to 1 in dimensionless units. The results presented below are not qualitatively changed by another reasonable value of $h_{\mathrm{s}}\left(h_{\mathrm{s}} \approx 0.5-2\right)$.

Equation (3) implies a renormalization of the stiffness $\xi_{\mathrm{c}}^{2}$ of the $|\nabla \Phi|^{2}$ term coming from the $|\nabla \Psi|^{2}$ term. The typical deformation energy of the concentration order parameter is $(1 / 2) k_{\mathrm{B}} T \xi_{\mathrm{c}}^{2}|\nabla \Phi|^{2}$ which is related to a typical free energy per helium atom of the order $k_{\mathrm{B}} T$. The typical superfluid free energy per atom is of order [19] $(1 / 2) E_{\mathrm{s}} \xi_{\mathrm{s}}^{2}|\nabla \Psi|^{2} / \rho_{0}$ where $E_{\mathrm{s}} \approx$ $(2 \pi)^{-1}\left(h^{2} / m^{* 2}\right) \rho_{0}$ and $\rho_{0}=|A|^{2}$ is the bare superfluid density. From the value of the universal superfluid jump $\rho_{\mathrm{s}} / T=4 \pi k_{\mathrm{B}} m^{* 2} / h^{2}[6,19]$, we estimate $E_{\mathrm{s}} \approx k_{\mathrm{B}} T\left(\rho_{0} / \rho_{\mathrm{s}}\right)$ which is larger than $k_{\mathrm{B}} T$. The gradient term is therefore of the form $(1 / 2) k_{\mathrm{B}} T \xi^{2}|\nabla \Phi|^{2}$ with $\xi$ given by

$$
\xi^{2}=\xi_{\mathrm{c}}^{2}+\left(E_{\mathrm{s}} / k_{\mathrm{B}} T\right) \xi_{\mathrm{s}}^{2} .
$$

Therefore, the concentration order parameter stiffness is controlled both by the atom-atom interaction and by the superfluid order. Expression (8) suggests that the superfluid contribution to this stiffness is not at all negligible but in fact brings in the dominant contribution.

In the following, all distances will be expressed in units of this characteristic length $\xi$ which we estimate to be in the range $0.5-2$ a.l. In the following, we take $\xi \approx 1$ a.l. Another choice rescales all lengths accordingly.

2.6 Global CONSERVATION. - The films are usually in equilibrium with a gas in a finite box. At $T=1.1 \mathrm{~K}$ and for a pressure $p \approx 10 \mathrm{~mm} \mathrm{Hg}$ (for $X=0.6)$ corresponding to the bulk liquid-gas equilibrium at this temperature, the ideal gas law yields a quantity of helium in the gas corresponding to less than 0.1 a.1. in the film [25]. This gives an upperbound for the amount of helium in the gas since typical experiments are carried on at lower temperatures and lower gas pressures.

Therefore, we may consider the films as closed systems and expression (2) must be supplemented by the condition of global conservation of the species. This is done via the introduction of a Lagrange parameter which can alternatively be seen as a bulk field $\lambda$ competing with the surface field $h_{\mathrm{s}}$. This additional field introduces a complication in the treatment and one has to resort to numerical computation to solve the Euler-Lagrange equations resulting from the extremalization of equation (2). This constraint is expressed mathematically by :

$$
\int_{0}^{d} \mathrm{~d} z \Phi(z)=0 \text {. }
$$


It changes the physics of the problem since it amounts to imposing a variable field. The corresponding problem of a thin fluid film without the constraint (9) i.e. in contact with a reservoir has been treated in [26]. In this case, one still predicts abrupt phase separations but displaced from the bulk transition towards lower temperatures at fixed concentration or inside the bulk phase separation diagram at fixed temperature with a displacement proportional to $1 / d$ which is characteristic of surface effects in the large $d$ limit. Furthermore, below a critical thickness, it is predicted that no phase separation occurs anymore [26]. In our case, with (9), we will obtain features resembling these results but rounded out by the presence of the variable bulk field $\lambda$.

2.7 EUlER-LAgrange EQUATIONS. - One has now to extremalize $\int_{0}^{d} \mathrm{~d} z[f\{\Phi(z)\}-\lambda \Phi(z)]$. We obtain the Euler-Lagrange equations

$$
\begin{aligned}
& \partial \Phi /\left.\partial z\right|_{z=0}=h_{\mathrm{s}} \\
& \partial \Phi /\left.\partial z\right|_{z=d}=0
\end{aligned}
$$

with the invariant integral

$$
\begin{gathered}
(\partial \Phi / \partial z)^{2}-G(\Phi)+\lambda \Phi=E \\
E=\left.(\partial \Phi / \partial z)^{2}\right|_{z=d}-G(\Phi(d))+\lambda \Phi(d) \\
=-G(\Phi(d))+\lambda \Phi(d)
\end{gathered}
$$

from equation (11). Setting

$$
\underline{G}(\Phi)=G(\Phi)-G(\Phi(d))+\lambda(\Phi-\Phi(d))
$$

and searching for increasing ${ }^{3} \mathrm{He}$ concentration profiles as a function of $z$, we get from (12)

$$
\partial \Phi / \partial z=\sqrt{\underline{G}(\Phi)}
$$

and the system of two integral equations for $\Phi(0)$ and $\Phi(d)$ :

$$
\begin{aligned}
& \int_{\Phi(0)}^{\Phi(d)} \mathrm{d} \Phi / \sqrt{\underline{G}(\Phi)}=d \\
& \int_{\Phi(0)}^{\Phi(d)} \Phi \mathrm{d} \Phi / \sqrt{\underline{G(\Phi)}}=0 .
\end{aligned}
$$

Equations (15) and (16) hold as long as the ${ }^{3} \mathrm{He}$ boundary concentration on the substrate obeys $\Phi(0) \leqslant 0$. This is verified for weak surface fields $h_{\mathrm{s}}$. For strong surface fields, one has to look for solutions in a larger class of profiles which can display discontinuities in the first derivative : in the resolution, as soon as $X(z)<0$, we project the concentration profile on the value $X=0$, corresponding to the existence of pure ${ }^{4} \mathrm{He}$ phase on the substrate. Equation (16) expresses the conservation of ${ }^{3} \mathrm{He}$ atoms.
Equations (10) and (12) yield the additional algebraic equation

$$
\begin{aligned}
G(\Phi(d))-G & (\Phi(0))- \\
& -\lambda(\Phi(d)-\Phi(0))+h_{\mathrm{s}}^{2}=0 .
\end{aligned}
$$

The three equations (15-17) must be solved for the three unknown parameters $\lambda, \Phi(0)$ and $\Phi(d)$. The resolution is carried on a computer with a grid optimization method [27]. The integrals are transformed into combinations of elliptic integrals of the first and third kinds which are calculated by series expansions [28]. Solving equations (15) and (16) yields $\Phi(0)$ and $\Phi(d)$ from which $\Phi(z)$ is calculated via equation (12). Then, relevant physical quantities such as the thermodynamic potential $\lambda$ can be calculated.

\section{Results.}

The results of our numerical study of equations (1517) for various mean ${ }^{3} \mathrm{He}$ concentrations and temperatures are summarized in figures 1-4. Note the following points :

1) the films are never completely homogeneous or stratified but present instead intermediate concentration profiles as shown in figures 1 and 2 . Very thin films display a very steep concentration profile due to the effect of the surface field which selects the ${ }^{4} \mathrm{He}$ on the substrate. For these very thin films, the bulk energy density $G(\Phi)$ plays almost no role and the corresponding profiles result from the competition between the gradient and the surface energies.

For increasing thicknesses, the profiles first become smooth (quasi-homogeneous regime) but they
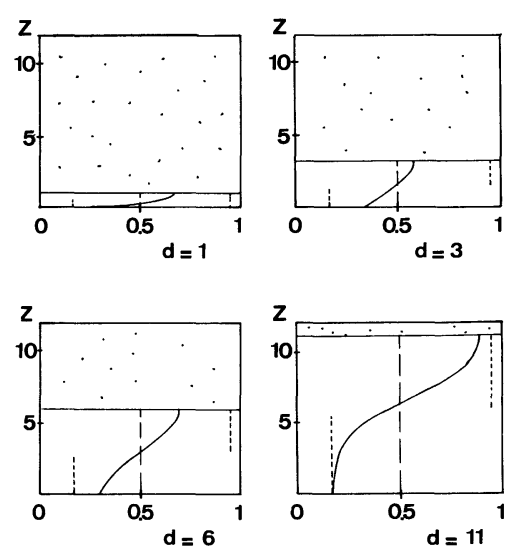

Fig. 1. - Evolution of the concentration profile as a function of the direction $z$ perpendicular to the film for several total thicknesses $d$ at fixed ${ }^{3} \mathrm{He}$ concentration depicted by the dotted-dashed line. $d$ and $z$ are given in units of the characteristic length $\xi$ introduced in paragraph 2.5. Typically, $\xi \approx 1$ a.1. The dashed lines indicate the values $X_{\mathrm{S}}(T)$ and $X_{\mathrm{N}}(T)$ of the bulk superfluid and normal phases. 

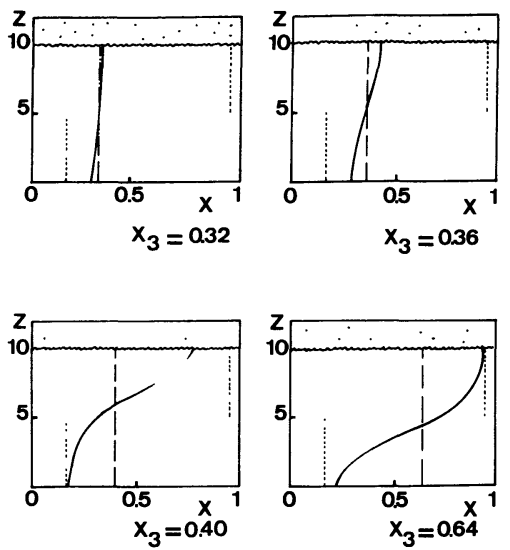

Fig. 2. - Evolution of the concentration profile $\Phi(z)$ at fixed total thickness $d / \xi=10$, for different mean ${ }^{3} \mathrm{He}$ concentrations from $X=0.32$ to 0.64 . The same convention as in figure 1 is used. Figure 2 shows that a small variation of the mean concentration leading to a very small change in bulk values $X_{\mathrm{S}}(T)$ and $X_{\mathrm{N}}(T)$ results in a drastic evolution of the concentration profile. The structure changes from almost homogeneous for $X_{0}=0.32$ to clearly stratified for $X_{0} \geqslant 0.42$.

sharpen again at higher thicknesses, appearing as stratified with a ${ }^{4} \mathrm{He}-{ }^{3} \mathrm{He}$ interface width of the order of $\xi$. This behaviour is shifted towards larger thicknesses as the surface field is made larger. Figure 2 shows that a small variation of the mean concentration leading to a very small change in bulk values $X_{\mathrm{S}}(T)$ and $X_{\mathrm{N}}(T)$ results in a drastic evolution of the concentration profile. It changes from almost homogeneous for $X_{0}=0.32$ to clearly stratified for $X_{0} \geqslant 0.42$;

2) by extension from the bulk case, one can speak of quasi-homogeneous films when $\Phi(0) \approx \Phi(d)$ and of quasi-stratified films when $\Phi(0)$ and $\Phi(d)$ are very different. This distinction is quantified in figure 3 which presents $X(z=0)$ and $X(z=d)$ as a function of the ${ }^{3} \mathrm{He}$ concentration $X_{0}=$ $d_{3} /\left(d_{3}+d_{4}\right)$, where $d_{3}$ and $d_{4}$ are the equivalent ${ }^{3} \mathrm{He}$ and ${ }^{4} \mathrm{He}$ thicknesses expressed in units of $\xi$, for different values of the total film thickness $d=d_{3}+d_{4}$.

In the limit $d \rightarrow+\infty$, one recovers the rigorously stratified structure inside the bulk coexistence region depicted in dotted-dashed lines forming the rectangle in figure 3. In the bulk case, the state is homogeneous outside the rectangle (in this case $\Phi(0)<$ $\Phi(d)=0$ due to a surface effect : as the coexistence curve is approached, a wetting layer of ${ }^{4} \mathrm{He}$ rich phase grows and leads to complete wetting at the coexistence point : see [29] for a discussion of this point) ;

3) in the case of films of finite thickness $d$, the two upper and lower curves settle the boundaries of the finite size coexistence domain: within this domain,

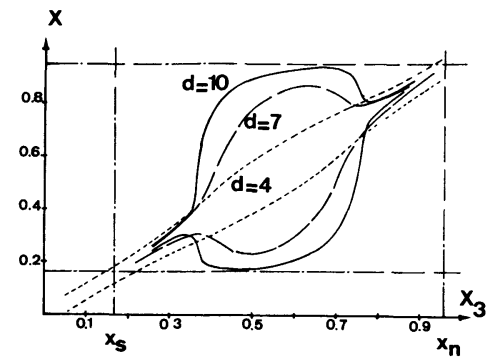

Fig. 3. - Representation of the ${ }^{3} \mathrm{He}$ concentration on the substrate (lower curve) and on the liquid-gas interface (upper curve) for different film thicknesses : $d / \xi=10$ (continuous curve), $d / \xi=7$ (large dashed curve), $d / \xi=4$ (small dashed curve). For each film thicknesses $d$, the upper and lower curves settle the boundaries of the finite size coexistence domain : inside this domain, the film exhibits a stratified structure with $\Phi(0) \ll \Phi(d)$ and outside a quasi-homogeneous state with $\Phi(0) \approx \Phi(d)$. The larger rectangle corresponds to the bulk concentration values : the domain of coexistence denoted by the lower and upper curves at a finite thickness $d$ should tend to the large rectangle in the limit $d \rightarrow+\infty$.

the film exhibits a stratified structure and outside a quasi-homogeneous state with $\Phi(0) \approx \Phi(d)$. Even for relatively large film thicknesses, important departures from the bulk case are observed : the domain of quasi-coexistence decreases as $d$ decreases and disappears for $d / \xi \leqslant 4$ (see Fig. 3) : this is reminiscent of the disappearance of the phase separation transition in films in contact with a reservoir [26] i.e. in the absence of a variable bulk field $\lambda$. In our case, true transitions no longer exist and the abrupt changes are rounded out by the variable field $\lambda$.

One would expect a transition shift $\Delta X_{3}$ which scales as $\Delta X_{3} \approx d^{-1}$ and a transition width $\delta X_{3}$ which scales as $\delta X_{3} \approx \mathrm{e}^{-d / \xi}$ in the limit $d \rightarrow+\infty$ resulting from finite size effects at first order phase transitions $[15,26]$ : the $\Delta X_{3} \approx d^{-1}$ law reflects the $d^{-1}$ relative weight of the surface terms compared to the volume term [26] and the $\delta X_{3} \approx \mathrm{e}^{-d / \xi}$ reflects the exponential relaxation of the concentration profile in the Landau-Ginzburg model [30,31] with the constraint of conservation of the helium. We have been unable to verify these asymptotic laws numerically due to numerical instabilities and prohibitive computation time for $d / \xi \geqslant 15-20$. Anyway, this shows that the range $d \leqslant 10 \xi$ is outside the asymptotic regime and is characterized by non-universal behaviours ;

4) we now compare the prediction of our model for the third sound velocity with experimental results. The third sound velocity scales as $[32,33]$ (see the appendix)

$$
C_{3}^{2} \approx \sigma_{\mathrm{s}} \partial \mu /\left.\partial z\right|_{z=d} .
$$

Here, we make the simplifying assumption that the superfluid areal density $\sigma_{s}$ is proportional to 
$d_{4}$ and neglect the variation of the superfluid density due to ${ }^{3} \mathrm{He}-{ }^{4} \mathrm{He}$ interactions. Expression (18) is valid for a homogeneous film. In the appendix, we develop a potential hydrodynamic approach which confirms the validity of (18) for arbitrary concentration profiles.

Usually, $\mu$ essentially contains the contribution of the Van der Waals substrate potential which scales as $\mu_{\mathrm{VdW}} \approx \theta d^{-3}$ where $\theta \approx 30-50 \mathrm{~K}$ [12]. Note that a consistent treatment should incorporate $\mu_{\mathrm{VdW}}$ in the free energy density (2). However, this leads to a much more involved analysis since the Euler-Lagrange equations are no more autonomous and no invariant integral is then available. Also, the expected corrections on the concentration profiles are small (see discussion of Ref. [29]). We therefore take the view that $\mu_{\mathrm{VdW}}$ has two distinct effects : 1) a bulk-field applied on the concentration profile (which we neglect in this respect except at $z=0$ with $h_{\mathrm{s}}$ ) and 2) a stiffness field acting on the upper interface and which we take explicitly into account for the third sound velocity.

In addition to $\mu_{\mathrm{VdW}}$, our model shows that another thermodynamic potential $\lambda$ appears and creates a restoring force in the third sound velocity. It stems from a thermodynamic stiffness of the film concentration profile. This second term is just $\lambda=\partial F / \partial \Phi$ and, from equation (17), it reads

$$
\begin{aligned}
\lambda=\{ & G(\Phi(d))- \\
& \left.-G(\Phi(0))+h_{\mathrm{s}}^{2}\right\} /(\Phi(d)-\Phi(0)) .
\end{aligned}
$$

Therefore, the total restoring force controlling the third sound velocity is

$$
\partial \mu /\left.\partial z\right|_{z=d}=\partial \lambda /\left.\partial z\right|_{z=d}+\partial \mu_{V d W} /\left.\partial z\right|_{z=d} .
$$

The additivity is of course an approximation but is justified by an estimation from a direct resolution of the differential equation in the presence of the full Van der Waals potentials [29]. The correction $\lambda$ to the $\mu_{\mathrm{VdW}}$ is of the order of $20 \%$ at $d \approx 8 \mathrm{a} .1$. and increases in relative weight as $d$ increases in this intermediate thickness range $3 \leqslant d \leqslant 10$. This does not mean that $\lambda$ has a longer range than the $d^{-3}$ Van der Waals potential but only that in a narrow range of thicknesses, an exponential dependence $\lambda \approx \mathrm{e}^{-d / \xi}$ can dominate.

The third sound velocity computed from equations (18) and (20) is represented in figure 4a in comparison with experimental results already published in [12]. For the sake of comparison, we have taken $\xi=1$ a.l., $\theta=50 \mathrm{~K}$ and $a \approx 2 \mathrm{~K}$ (the same curve is obtained with $\theta=25 \mathrm{~K}$ and $a \approx 1 \mathrm{~K})$. Note the excellent agreement which is not very sensitive to the values of the parameters : changing $a(T)$ from $1 \mathrm{~K}$ to $2 \mathrm{~K}$ with $\theta=50 \mathrm{~K}$ does not shift the dotted line by more than $10 \%$ of the difference between the continuous and dashed curves. The continuous line

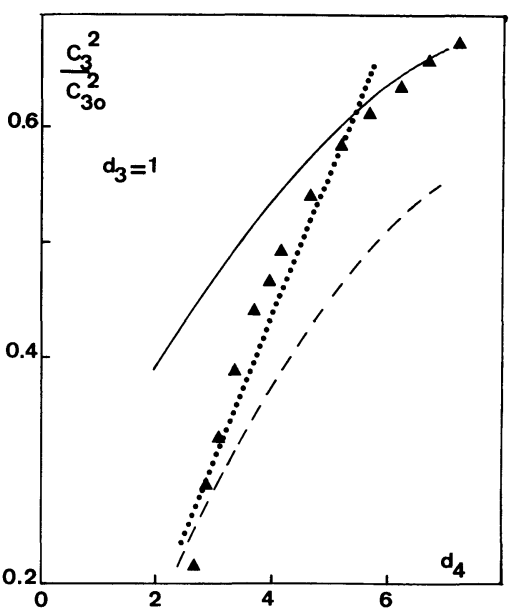

a)

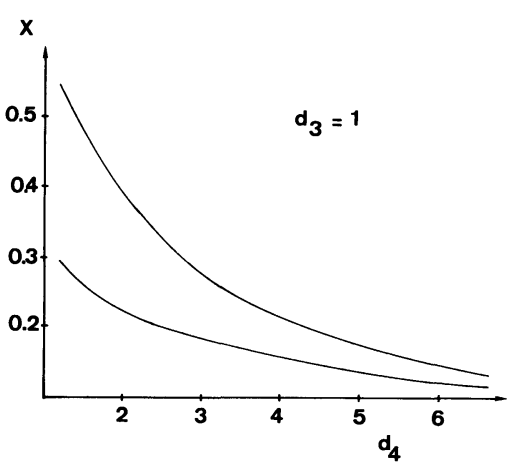

b)

Fig. 4. - The third sound velocity computed from equations (18) and (20) is represented in figure 4a (dotted line) along with experimental results (triangles) already published in [12]. For the sake of comparison, we have taken $\xi=1$ a.l. The continuous line is the prediction of the Guyer-Miller hydrodynamic model in a rigorously stratified film and the dashed line is the prediction for a perfectly homogeneous film. The apparent continuous transition from the dashed curve to the continuous curve has led the authors of [12] to suggest that the film was undergoing a homogeneous-stratified transition. In figure $4 \mathrm{~b}$, we represent the corresponding variation of the ${ }^{-3} \mathrm{He}$ concentration on the substrate (lower curve) and at the liquid-gas interface (upper curve). Since the system representation point is outside the finite size coexistence domain shown in figure 3 , the film structure is not stratified. This suggests that in this case the evolution of the third sound velocity is not associated with a qualitative change of the structure of the film from homogeneous to stratified.

is the prediction of the Guyer-Miller hydrodynamic model [34] in a rigorously stratified film and the dashed line is the prediction for a perfectly homogeneous film. The apparent continuous transition from the dashed to the continuous curve has led the authors of [12] to suggest that the film was undergoing a homogeneous-stratified transition. 
In figure $4 \mathrm{~b}$, we represent the corresponding variation of the ${ }^{3} \mathrm{He}$ concentration on the substrate (lower curve) and at the liquid-gas interface (upper curve). Since the system representation point is outside the finite size coexistence domain shown in figure 3, the film structure is not stratified. This suggests that the evolution of the third sound velocity is not associated, in this case, with a qualitative change of the structure of the film from homogeneous to stratified. In fact, the third sound probe appears to be extremely sensitive to the film structure via the field $\lambda$ (see the appendix) and its variations amplify the small changes in the concentration profile. Figure 5 shows the same types of results as in figure $4 \mathrm{a}$ but for $d_{3}=2 \mathrm{a}$.1. The agreement with experimental results is less striking but the trend is good;

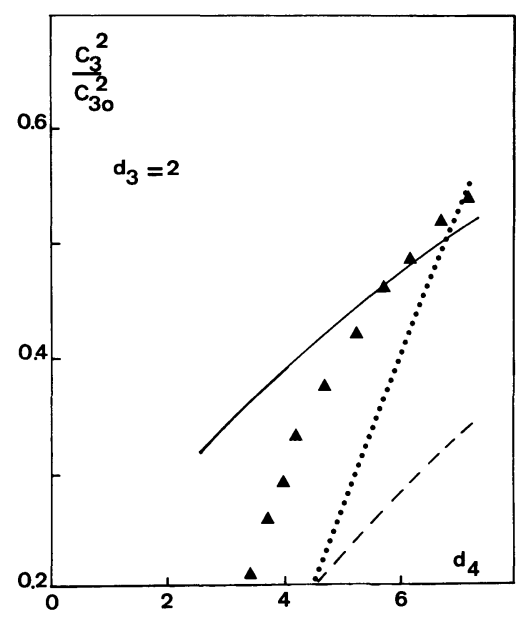

Fig. 5. - Similar results as in figure 4 but for $d_{3}=2$ a.l.

5) recent experiments [35] have studied the evolution of the film structure upon addition of ${ }^{3} \mathrm{He}$ at constant $d_{4} \approx 3$ a.l. Measures of superfluidity onset temperatures $T_{0}$ indicate that as $d_{3}$ increases, $T_{0}$ decreases asymptotically towards a constant value which is just the onset superfluid temperature measured by McQueeny et al. [5] for the same amount of ${ }^{4} \mathrm{He}$ but loaded with 12 a.l. of ${ }^{3} \mathrm{He}$. This suggests that the mixture films are stratified for $d_{3} \geqslant 4$ and that addition of ${ }^{3} \mathrm{He}$ beyond four atomic layers does not affect the superfluid phase.

We have tested these results within our model: figure 6 shows the computed variation of the ${ }^{3} \mathrm{He}$ concentration on the substrate (lower curve) and at the liquid-gas interface (upper curve) at constant ${ }^{4} \mathrm{He}$ coverage and at increasing ${ }^{3} \mathrm{He}$ thicknesses $d_{3}$. Figure 6 clearly shows a smooth transition from a homogeneous state $(\Phi(d) \approx$ $\Phi(0))$ to a stratified state where the substrate and liquid-gas interface concentrations are very different,

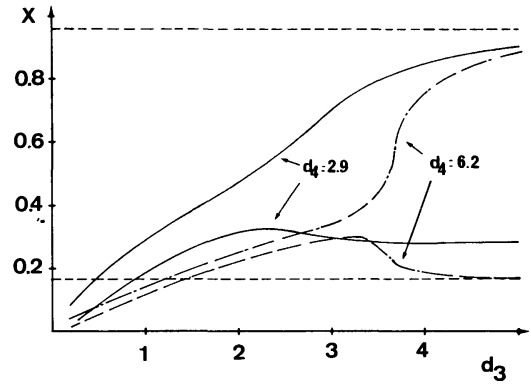

Fig. 6. - Variation of the ${ }^{3} \mathrm{He}$ concentration on the substrate (lower curve) and at the liquid-gas interface (upper curve) at constant ${ }^{4} \mathrm{He}$ coverage and at increasing ${ }^{3} \mathrm{He}$ thicknesses $d_{3}$ computed within our model. It clearly shows a smooth transition from a homogeneous $(\Phi(d) \approx \Phi(0))$ to a stratified state where the substrate and liquid-gas interface concentrations are very different, at a value $d_{3}$ of roughly four atomic layers. The computation has been made with the same values of the parameters as for the other figures.

at a value $d_{3}$ roughly around four atomic layers. The computation has been made with the same values of the parameters as for the other figures. Note that the transition is better defined for larger ${ }^{4} \mathrm{He}$ coverages. Figure 6 can be interpreted from figure 3 as the penetration of the configuration point inside the finite-size coexistence domain;

6) temperature effects may also be studied within our approach. Figure 7 represents the variation of the ${ }^{3} \mathrm{He}$ concentration on the substrate (lower curve) and at the liquid-gas interface (upper curve) at constant ${ }^{4} \mathrm{He}$ and ${ }^{3} \mathrm{He}$ coverages $\left(d_{4}=6.4\right.$ and $\left.d_{3}=3.6\right)$ as a function of the temperature. In our computations, we have kept constant the coefficient $a$ in equation (5). The effect of temperature appears

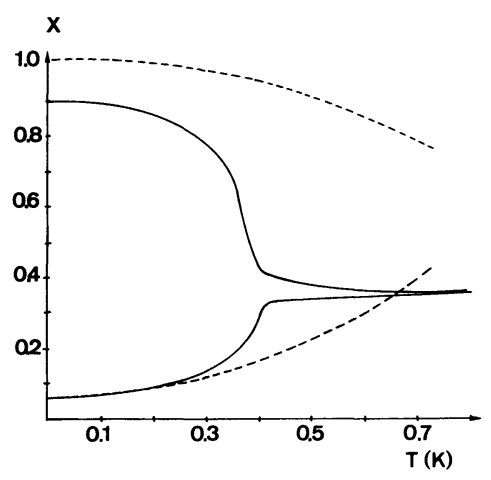

Fig. 7. - Variation of the ${ }^{3} \mathrm{He}$ concentration on the substrate (lower curve) and at the liquid-gas interface (upper curve) at constant ${ }^{4} \mathrm{He}$ and ${ }^{3} \mathrm{He}$ coverages $\left(d_{4}=6.4\right.$ and $d_{3}=3.6$ ) as a function of temperature. In our computations, we have kept constant the coefficient $a$ in equation (5). The effect of temperature appears only via the variation of the values of the bulk concentrations $X_{\mathrm{S}}(T)$ and $X_{\mathrm{N}}(T)$. 
only via the variation of the values of the bulk concentrations $X_{\mathrm{S}}(T)$ and $X_{\mathrm{N}}(T)$. The continuous curves show a homogeneous-stratified transition around $T=0.35 \mathrm{~K}$ whereas the bulk values presented by the dashed lines exhibit only a very smooth behaviour. These results are reminiscent of the experimental finding of a sharp increase of the third sound velocity as $T$ decreases [35].

\section{Discussion.}

We have presented a simple model for the homogeneous-stratified transitions observed in ${ }^{3} \mathrm{He}-{ }^{4} \mathrm{He}$ binary mixture films using the third sound technique. A continuous Landau-Ginzburg model with a bulk term containing both atom-atom and superfluid contributions plus surface and interface terms with, in addition, the constraint of conservation of helium 3 and helium 4 has been compared with experiments. Our most important results are the following :

1) compared to previous models [12], our approach has suggested precise explicit pictures for the different film structures (via the $\Phi(z)$ field) deduced from the analysis of experiments;

2) it has stressed the intricate interplay between bulk, surface and interface energies as occurs in this range of thicknesses ( 3 atomic layers $\leqslant$ $d \leqslant 20$ atomic layers). The behaviour of these films therefore appears to be intermediate between two and three dimensions and points at the importance of finite size effects ;

3) the important role of the bare superfluid order has been recognized to control largely the stiffness of the ${ }^{3} \mathrm{He}$ concentration profile in the direction perpendicular to the substrate. This is in the spirit of the discussion of [36]. In usual normal fluids, the same qualitative results should hold but for smaller thicknesses $\left(d / \xi_{c}\right)$ due to the absence of the superfluid stiffness;

4) our results have suggested that a steep change in the third sound velocity (which can be obtained experimentally) is not necessarily connected with a corresponding sharp evolution of the concentration profile. In other words, the abrupt increase or decrease of the third sound velocity is not the unambiguous signature of a film phase transition. This stems from the great sensitivity of the third sound probe which amplifies the evolution of concentration profiles ;

5) the third sound velocity depends continuously on the film structure via the value of the thermodynamic potential. Therefore, the dichotomic choice stratified or homogeneous imposed by the use of the Guyer-Miller model [34] has been refined in the appendix to take into account smooth concentration profiles with in fine the validation of the
Atkins-Rudnick formula [33]. The case when a pure normal phase floats above the superfluid in very inhomogeneous films remains an open problem to tackle in full generality ;

6) the connection between the present approach and the previous multilayer interface model (MIM) presented in [12] to account for the third sound experiments is similar in spirit to the unification of the capillary and Van der Waals view points for describing a single interface. More precisely, the MIM essentially weighs the mixing entropy which is taken into account in the present approach by the gradient term in the Landau-Ginzburg expansion for the chemical potential. Our present approach has the advantage of clearly relating the physics in thin ${ }^{4} \mathrm{He}-{ }^{3} \mathrm{He}$ mixture films to the known surface and finite size physics and moreover allows quantitative predictions.

Let us end by a list of the limitations and possible extensions.

As a first extension, it would be useful to treat the full Hamiltonian (2) with the two order parameters $\Phi$ and $\Psi$. Furthermore, our interdimensional treatment does not include the coupling with the twodimensional Kosterlitz-Thouless superfluidity. This could be done using the tools developed in [37, 38]. Indeed, Berker and Nelson [37] have used a vectorial generalization of the Blume-Emery-Griffiths model which have been solved using an approximate Migdal-Kadanoff renormalization scheme. This model correctly embodies the Kosterlitz-Thouless nature of the superfluidity, the $\mathrm{He}-\mathrm{He}$ interactions and of the coupling between superfluidity and concentration. However, it is essentially two-dimensional and does not take into account the strong influence of the substrate and of the liquid-gas surfaces. The superficial superfluid transition of a ${ }^{3} \mathrm{He}-{ }^{4} \mathrm{He}$ bulk mixture in contact with a wall attracting preferentially the ${ }^{4} \mathrm{He}$ (semi-infinite geometry) has also been studied with the same tools [4]. It would be desirable to extend the analysis to the finite film case. Mon and Saam [38] have gone a step in this direction by considering a two-layer ${ }^{3} \mathrm{He}-{ }^{4} \mathrm{He}$ mixture model and have studied the consequences of departing from a purely two-dimensional framework including the effect of the substrate potential. Compared to the experiments presented in [12], the discreteness of the ${ }^{3} \mathrm{He}$ levels does not seem to be relevant and furthermore, the experiments were performed at an essentially constant amount of ${ }^{4} \mathrm{He}$ and ${ }^{3} \mathrm{He}$ and not at a fixed chemical potential. The general treatment of superfluid films remains an interesting question for future investigations.

As $T$ decreases towards absolute zero, coupling of the third sound with the sound in the gas above the film can become large and may drastically change the interpretation of the third sound experiments 
and therefore the relevant comparison with our slab model [39].

Improvements in the presented model will be carried on in a future work along the following lines: 1) one should take the $z^{-3}$ substrate Vander Waals potential into account completely (this implies tackling non-autonomous second-order differential equations), 2) one should treat the competition between the two order parameters $\Phi$ and $\Psi$, explicitly within the Landau-Ginzburg formalism and 3) finally one should address the KosterlitzThouless nature of the superfluidity in addition to all other features.

\section{Appendix.}

The technique of the third sound velocity measurements has evolved as a very efficient and sensitive probe for testing the structure and the physical properties of thin superfluid films. Third sound is similar to a gravity shallow wave. Its velocity is therefore a function of the film thickness $d$ as $C_{3} \approx(g d)^{1 / 2}[32,33]$, and of its concentration profile (stratified or homogeneous) via the value of the effective spring force $g$ which is simply the gradient of the fluid chemical potential. When the film is heterogeneous, $g$ is changed. Direct measurement of $C_{3}$ gives access to the physical state of the film via the modelling process film structure $\rightarrow C_{3}$.

The problem of determining the structure of a film from a third sound experiment is therefore to invert this film structure $\rightarrow C_{3}$ transformation. However, this inversion is impossible to perform in its largest generality since it is related to the general class of inverse problems where a function characterizing the film structure (namely the superfluid concentration profile $\Phi(z)$ ) must be recovered from the knowledge of a single number $\left(C_{3}\right)$. The only way to get information on $\Phi(z)$ from the measurement of $C_{3}$ is to use a priori knowledge in order to restrict the space of possible functions compatible with a given measurement. This is done usually via models which achieve simple connections between structure and third sound velocity.

At present, there exists only one such film structure $\rightarrow C_{3}$ model. It is the hydrodynamical treatment of the third sound propagation of Guyer and Miller [34] which considers two extreme cases : only the completely uniform film and the completely stratified film in which each layer contains a completely homogeneous fluid.

In order to improve information recovery in the comparison between theoretical predictions for the films structures obtained in models with experimental results, we propose a simple hydrodynamical model of the third sound velocity in inhomogeneous ${ }^{4} \mathrm{He}-{ }^{3} \mathrm{He}$ mixture films which generalizes the GuyerMiller approach to encompass the case of continuous superfluid concentration profiles.
We start from the usual linearized equations of motion of the two-fluid model, for ${ }^{4} \mathrm{He}-{ }^{3} \mathrm{He}$ mixtures, supplemented with the following assumptions: i) the liquid film is assumed to be incompressible, ii) we neglect the interactions of the film with the vapor and therefore any coupling with modes in the gas, iii) we assume that there is neither horizontal nor vertical motion of the normal fluid component, i.e. the normal fluid is clamped. This approximation results from the damping effect of the viscosity in the motion of the normal fluid as estimated by comparing the film thickness $d$ with the viscous penetration length $\delta(d \ll \delta)$. This assumption allows us to simplify the hydrodynamic model considerably by getting rid of the momentum and entropy conservation equations in which one should take the viscous and diffisivity terms into account. One can imagine the motion of the superfluid component as invading the free space left by the normal component in a way similar to the motion of a superfluid in a porous medium. This assumption should be valid if the superfluid invades more or less the whole available film thickness. However, should it not be the case (as for example when a layer of normal fluid floats on the superfluid), the treatment should then incorporate the pressure effect induced by the presence of the dead ${ }^{3} \mathrm{He}$ layer which has been termed blanket effect [34]. This is done in our treatment by a suitable definition of the chemical potential $\mu$. Finally note that a consequence of the clamping of the normal fluid is the attenuation of third sound. We do not try to describe or extract information from the attenuation data [33].

\section{The generalized hydrodynamic model.}

The two basic hydrodynamic equations therefore read

$$
\begin{gathered}
\operatorname{div}\left(\rho_{\mathrm{s}} \mathbf{v}_{\mathrm{s}}\right)=0 \\
\partial \mathbf{v}_{\mathrm{s}} / \partial t=-\nabla \mu
\end{gathered}
$$

Equation (A.1) is the mass conservation condition with the hypothesis of incompressibility and the condition $\mathbf{v}_{\mathrm{n}}=0 . \mathbf{v}_{\mathrm{s}}$ and $\mathbf{v}_{\mathrm{n}}$ are the superfluid and normal velocities respectively. Equation (A.2) is the superfluid equation which writes that the superfluid component can be accelerated by a gradient of chemical potential resulting either from a thermodynamic cause such as a pressure or temperature gradient or from any external potential such as gravity or Van der Waals interaction. From equation (A.2), one deduces that $\nabla \times \mathbf{v}_{\mathrm{s}}$ can be chosen zero. Therefore, $\mathbf{v}_{\mathrm{s}}$ becomes :

$$
\mathbf{v}_{\mathrm{s}}=\nabla \varphi
$$

where $\varphi$ is the scalar velocity potential, a function of the spatial components. Using (A.3) in (A.2) yields

$$
\partial \varphi / \partial t+\mu=0 \text {. }
$$


Consider the case of a film of thickness $d$ contained between $z=0$ and $z=d$ and a perturbation from its horizontal equilibrium state parametrized by the height profile $\zeta(x, y)$, where the plane $(x, y)$ is parallel to the substrate. In homogeneous fluids, we usually have $\partial \zeta / \partial t=v_{\mathrm{sz}}=\partial \varphi / \partial z$. Writing this relation for an inhomogeneous fluid would imply that a motion $\zeta$ of the liquid-gas interface does not involve any motion of the normal component of the fluid. This is not correct since we cannot distinguish which fluid is convected by a vertical motion of the interface. The generalized relation between $\zeta$ and $v_{z}$ at $z=d$ is obtained as follows. We define a surface $S\left(\gg \zeta^{2}\right)$ on the liquid-gas interface. The fluid flux convected by the vertical motion of the interface is $S \rho(d) \partial \zeta / \partial t$ where $\rho=\rho_{\mathrm{s}}+\rho_{\mathrm{n}}$ is the total fluid density. This flux is fed by the flowing in of superfluid flux $S \rho_{\mathrm{s}} v_{\mathrm{sz}}(d)$ where $v_{\mathrm{s} z}$ is the component of $\mathbf{v}_{\mathrm{s}}$ along the vertical axis $z$. Equating the two terms yields the generalized boundary condition at the free interface :

$$
\rho(d) \partial \zeta / \partial t=\rho_{\mathrm{s}}(d) v_{\mathrm{s} z}(d) .
$$

Let us write equation (A.4) at the liquid-gas interface. In the presence of the disturbance $\zeta$, it is convenient to translate equation (A.4) from $z=d+\zeta$ to $z=d$. Therefore, equation (A.4) becomes :

$$
\partial \varphi / \partial t+\mu(d)+\zeta \partial \mu / \partial z=0
$$

which by time derivation and use of equation (A.5) gives

$$
\begin{array}{r}
\partial^{2} \varphi / \partial t^{2}+(\partial \mu / \partial z)\left(\rho_{\mathrm{s}}(d) / \rho(d)\right) \partial \varphi / \partial z=0 \\
\text { at } z=d .
\end{array}
$$

We can explicit equation (A.1) under the form :

$$
\rho_{\mathrm{s}} \operatorname{div} \mathbf{v}_{\mathrm{s}}+v_{\mathrm{s} z} \partial \rho_{\mathrm{s}} / \partial z=0 .
$$

In equation (A.1) we have used the fact that the density $\rho_{\mathrm{s}}$ is only a function of $z$ since the film is stratified only in the $z$ direction. Using equation (A.3) in (A.8) gives

$$
\rho_{\mathrm{s}} \nabla^{2} \varphi+\left(\partial \rho_{\mathrm{s}} / \partial z\right) \partial \varphi / \partial z=0 .
$$

Equations (A.7) and (A.9) are the two basic equations for describing the third sound propagation in an inhomogeneous film. They must be supplemented by the condition that the vertical fluid velocity $v_{z z}$ is zero on the substrate :

$$
\left.v_{\mathrm{Sz}}\right|_{z=0}=\partial \varphi /\left.\partial z\right|_{z=0}=0 .
$$

For homogeneous films $\left(\partial \rho_{\mathrm{s}} / \partial z=0\right)$, we recover from (A.7), (A.9) and (A.10) the well-known equations for the third sound propagation [33].

\section{Resolution of the hydrodynamic equations in inhomogeneous films.}

Note that the two equations (A.7) and (A.9) are identical to that of classical shallow waves in very inhomogeneous film fluids. The role of gravity is played by $(\partial \mu / \partial z)_{z=d}$. The theory of waves in heterogeneous liquids has been developed many years ago (see Ref. [40] for a general treatment) but only in the commonly encountered case of small heterogeneities characterized by a typical scale of the inhomogeneity variation (scale over which the density changes appreciably) larger than the wavelength of the excitation. In our case, we are in the other limit since the scale of the inhomogeneity variations is the film thickness $d$ which is of the order of a few atomic layers. This is very small compared to the third sound wavelength $\approx 1 \mathrm{~cm}$. Therefore, equations (A.7) and (A.9) have not been analysed previously in the literature : ${ }^{4} \mathrm{He}-{ }^{3} \mathrm{He}$ ultra-thin film mixtures provide one of the few opportunities to observe such large heterogeneities at such small scales.

Resolution of equation (A.9) is well known in the context of shallow gravity waves. We search for a solution for $\varphi$ under the parametrization

$$
\varphi(x, z)=f(z) \cos (k x-\omega t) .
$$

Without loss of generality, we choose the direction of propagation in the $x$ direction. Using (A.11) in (A.7), (A.9) and (A.10), we obtain the following set of equations :

$$
\begin{array}{r}
\mathrm{d}^{2} f / \mathrm{d} z^{2}-k^{2} f+(\varepsilon(z) / d) \mathrm{d} f / \mathrm{d} z=0 \\
\mathrm{~d} f / \mathrm{d} z=0 \quad \text { at } \quad z=0 \\
\left(\rho_{\mathrm{s}}(d) / \rho(d)\right) \mathrm{d} f / \mathrm{d} z-\omega^{2} f / g=0 \text { at } z=d .
\end{array}
$$

We have defined the effective acceleration $g \equiv(\partial \mu / \partial z)_{z=d}$ and the dimensionless function

$$
\varepsilon(z)=\left(d / \rho_{\mathrm{s}}(z)\right) \cdot \mathrm{d} \rho_{\mathrm{s}} / \mathrm{d} z .
$$

The case $\varepsilon(z)=0$ yields the usual shallow wave equations resulting in the dispersion relation [33]

$$
\omega / k=(g d)^{1 / 2}
$$

since then $f(z)=\operatorname{ch} k z$ and

$$
\mathrm{d} f /\left.\mathrm{d} z\right|_{z=d}=k \operatorname{sh} k z \approx k^{2} z .
$$

More generally, one can solve the system of equations (A.12-14) for the dispersion relation $\omega(k)$ in the following way. The crucial step is to recognize that $k^{2} f \ll d^{-1} \mathrm{~d} f / \mathrm{d} z \quad$ i.e. $(k d)^{2} \ll d(d \log f / \mathrm{d} z)$, due to the extreme thinness of the film compared to the third sound wavelength $2 \pi / k$. This hypothesis will be verified consistently 
at the end. This allows to replace the term $k^{2} f$ by $k^{2}$ (with $f \approx 1$ ) in (A.12) which yields the solution

$$
\mathrm{d} f / \mathrm{d} z=\left[k^{2} / \rho_{\mathrm{s}}(z)\right] \int_{0}^{z} \mathrm{~d} z^{\prime} \rho_{\mathrm{s}}\left(z^{\prime}\right) .
$$

Expression (A.17) satisfies the first boundary condition (A.13). Inserting in equation (A.14), we obtain the following dispersion relation $(\omega / k)^{2}=$ $g d \cdot\left[\rho_{\mathrm{s}}(d) / \rho(d)\right]\left\{\rho_{\mathrm{s}}(d)\right\}^{-1} d^{-1} \int_{0}^{d} \mathrm{~d} z^{\prime} \rho_{\mathrm{s}}\left(z^{\prime}\right)$, which gives the third sound velocity

$$
C_{3}^{2}=g d\left\langle\rho_{\mathrm{s}}\right\rangle / \rho(d)
$$

where the average superfluid density is

$$
\left\langle\rho_{\mathrm{s}}\right\rangle=d^{-1} \int_{0}^{d} \mathrm{~d} z^{\prime} \rho_{\mathrm{s}}\left(z^{\prime}\right) .
$$

Equation (A.18) has the form of the Atkins and Rudnick equation [34]. The hydrodynamic potential theory justifies the use of equation (A.18) even for extremely inhomogeneous films.

We can now verify the assumption that $k^{2} f \ll d^{-1} \mathrm{~d} f / \mathrm{d} z$. Indeed, it is easy to obtain an upper bound for $\mathrm{d} f / \mathrm{d} z$ since $\int_{0}^{z} \mathrm{~d} z^{\prime} \rho_{\mathrm{s}}\left(z^{\prime}\right) \approx z\left\langle\rho_{\mathrm{s}}\right\rangle$ and $\int_{0}^{z} \mathrm{~d} u \int_{0}^{u} \mathrm{~d} z^{\prime} \rho_{\mathrm{s}}\left(z^{\prime}\right) \approx z^{2}\left\langle\rho_{\mathrm{s}}\right\rangle$, typically. Therefore, $\int_{0}^{z} \mathrm{~d} z^{\prime} \mathrm{d} f / \mathrm{d} z$ is of the order of $(k d)^{2}$ which is very small $\left(k d \approx 10^{-6}\right)$ which proves the validity of the assumption $f \approx 1$.

Note that in the case when the upper liquid-gas interface is pure ${ }^{3} \mathrm{He}$, the previous theory breaks down. One has to consider the ${ }^{4} \mathrm{He}-{ }^{3} \mathrm{He}$ instead of the liquid-gas interface and take into account the floating ${ }^{3} \mathrm{He}$ layer by adding to the chemical potential $\mu$ the pressure exerted by the ${ }^{3} \mathrm{H}$ layer and created by the ${ }^{3} \mathrm{He}$-substrate Van der Waals attraction. A hydrodynamical treatment of this blanket effect has been presented in [34] but a direct potential theory for general inhomogeneous fluids remains a challenging open problem.

\section{Acknowledgments.}

It is a pleasure to thank J. P. Laheurte, J. C. Noiray, L. Peliti and especially J. P. Romagnan for stimulating discussions.

\section{References}

[1] De Gennes, P. G., Rev. Mod. Phys. 57 (1985) 827.

[2] Binder, K., Critical behaviour at surfaces, in Phase transitions and critical phenomena, eds. C. Domb and J. L. Lebowitz (Academic Press) 8 (1983).

[3] Evans, R. and Tarazona, P., Phys. Rev. Lett. 52 (1984) 557.

[4] Crisanti, A. and Peliti, L., J. Phys. A 18 (1985) L543.

[5] McQueeny, D., Agnolet, G. and Reppy, J. D., Phys. Rev. Lett. 52 (1984) 1325 ;

Webster, E., Webster, G. and Chester, M., Phys. Rev. Lett. 42 (1979) 243.

[6] Kosterlitz, J. M. and Thouless, D. J., J. Phys. C 6 (1973) 1181.

Nelson, D. R. and Kosterlitz, J. M., Phys. Rev. Lett. 35 (1977) 1201.

[7] Bhattacharyya, B. and Gasparini, F. M., Phys. Rev. Lett. 49 (1982) 919.

[8] Sherrill, D. S. and Edwards, D. O., Phys. Rev. B 31 (1985) 1338.

[9] Papoular, M. and Romagnan, J. P., Europhys. Lett. 3 (1987) 839.

[10] Valles, J. M., Higley, R. H., Johnson, B. R. and Hallock, R. B., Proc. 18th int. Conf. on low temp. Phys., Kyoto 1987, Jpn. J. Appl. phys. 26, Suppl. 26-3 (1987) 287 ;

ROMAGNAN, J. P., private communication.

[11] Wang, X. W. and Gasparini, F. M., Phys. Rev. B 34 (1986) 4916.
[12] Laheurte, J. P., Noiray, J. C., Romagnan, J. P. and Sornette, D., J. Phys. France 47 (1985) 39 ; Phys. Rev. Lett. 53 (1985) 2421.

[13] Ellis, F. M., Hallock, R. B., Miller, M. D. and GuYer, R. A., Phys. Rev. Lett. 46 (1981) 1461.

[14] Valles, J. M., Smith, D. T. Jr. and Hallock, R. B., Phys. Rev. Lett. 54 (1985) 1528.

[15] BARber, M. N., Finite size scaling, in Phase transitions and critical phenomena, eds. C. Domb and J. L. Lebowitz (Academic Press) 8 (1983).

[16] Eddington, A., Superfluidity in restricted geometries, in The liquid helium, eds. J. G. M. Armitage and I. E. Farquhar (Academic Press) 1975.

[17] Gilabert, A., Ann. Phys. Fr. 2 (1977) 203-252.

[18] Ginzburg, V. L. and Pitaevskil, L. P., Sov. Phys. JEPT 34 (1958) 858.

[19] Minnhagen, P., Rev. Mod. Phys. 59 (1987) 1001.

[20] One could expect $|\Psi|^{2}=1-\Phi$ which expresses that the superfluid phase is pure ${ }^{4} \mathrm{He}$ and the normal phase is pure ${ }^{3} \mathrm{He}$. However, the existence of boundaries, which influence the two order parameters in a different way (increase of the ${ }^{4} \mathrm{He}$ concentration near the substrate due to zero point motion in presence of the substrate attracting Van der Waals potential but decrease of the superfluid order parameter due to proximity effects [16-18]), lead to the expectation that the relation $|\Psi|^{2}=1-\Phi$ does not hold in all regions of the film. As a consequence, the most natural step is to introduce an additional energy 
associated with the mismatch $1-\Phi-|\Psi|^{2}$ which is generically a quadratic term of the form $1 / 2 . \alpha\left\{1-\Phi-|\Psi|^{2}\right\}^{2}$, where $\alpha$ is a rigidity constant. In the limit $\alpha \rightarrow+\infty$, one recovers $|\Psi|^{2}=1-\Phi$. The choice of a finite $\alpha$ allows the two order parameters to decouple somewhat. Developing this quadratic energy, we obtain terms which can be incorporated in a redefinition of the free energy $G\left(\Phi,|\Psi|^{2}\right)$, with in addition, the last term in the r.h.s. of equation (2) embodying the degree of coupling between the two order parameters. Note that the form $\Phi .|\Psi|^{2}$ of the coupling can also be viewed as resulting from a mass term associated with gradients of the superfluidity different from $\left(h^{2} / 8 \pi^{2} m^{* 2}\right)$ and proportional to the ${ }^{3} \mathrm{He}$ concentration (L. Peliti, private communication).

[21] PAPOUlAR, M., private communication.

[22] Near the tricritical $\lambda$ point, $\Phi-\Phi_{\mathrm{S}}$ and $\Phi-\Phi_{\mathrm{N}}$ behave like $\left|T-T_{\lambda}\right|^{\beta}$ since they are proportional to the order parameter (and $\beta$ is the order parameter critical exponent). This implies, from equation (5), that $G(\Phi) \approx\left|T-T_{\lambda}\right|^{4 \beta}$. This scaling is in agreement with the canonical scaling form $G(\Phi) \approx\left|T-T_{\lambda}\right|^{2-\alpha}$ if $4 \beta=$ $2-\alpha$. This is verified in mean field theory in which $\alpha=0$ and $\beta=1 / 2$. Therefore, within our mean field treatment, the choice (5) is coherent.

[23] The choice of (6) corresponds to replacing the Edwards potential of a ${ }^{3} \mathrm{He}$ atom in a film as a function of $z$ by a delta function defined at $z=0$. The Edwards potential exhibits a deep sink in the proximity of the substrate and shows a complex structure away from $z=0$ with a much smaller amplitude as a result of the quantization of the ${ }^{3} \mathrm{He}$ energy levels. The approximation (6) therefore neglects these weak ${ }^{3} \mathrm{He}$ bound states. Although the influence of the Van der Waals interaction is felt a long distance away from the substrate, the local field $h_{\mathrm{s}}$, which is coupled with the order parameter on the substrate, is expected to embody the major contribution of this interaction.

[24] Kroll, D. M. and Lipowsky, R., Phys. Rev. B 28 (1983) 6435.

[25] Noiray, J. C. and Romagnan, J. P., private communication.
[26] Lipowsky, R. and Gompper, Phys. Rev. 29 (1984) 5213

Sornette, D., Phys. Rev. B 31 (1985) 4672.

[27] Data reduction and error analysis for the physical sciences, BeVington, P. R., Grashteyn, I. S. and RYZHIK, I. M., Table of integrals and products (Academic press) 1965.

[28] Byrd, P. F. and Friedman, M. D., Handbook of elliptic integrals for engineers and scientists (Springer-Verlag) 1971.

[29] Sornette, D. and Laheurte, J. P., J. Phys. France 47 (1986) 1951.

[30] Privman, V. and Fisher, M. E., J. Stat. Phys. 33 (1983) 385 ;

Fisher, M. E. and Berker, A. N., Phys. Rev. B 26 (1982) 2507.

[31] Cardy, J. L. and Nightingale, P., Phys. Rev. B 27 (1983) 4256.

[32] Bergman, D., Phys. Rev. 188 (1969) 370 ; Phys. Rev. A 3 (1971) 2058.

[33] Rudnick, I., Physical acoustics at UCLA in the study of superfluid helium in Proc. Int. School of Phys. Enrico Fermi Varenna : New directions in Physical acoustics, Ed. D. Sette 63 (1976) 112 ;

Atkins, K. R. and Rudnick, I., Prog. Low Temp. Phys., Ed. C. J. Gorter (North Holland, Amsterdam) 6 (1970).

[34] Guyer, R. A. and Miller, M. D., Phys. Rev. B 25 (1982) 5749 ;

Guyer, R. A., Phys. Rev. B 31 (1985) 2713.

[35] Romagnan, J. P., Laheurte, J. P., Noiray, J. C. and Papoular, M., Phys. Rev. B 22 (1988) 5171.

[36] Laheurte, J. P., Noiray, J. C., Romagnan, J. P. and Papoular, M., Proc. 18th Int. Conf. on Low Temperature Physics Kyoto (1987), Jpn. J. Appl. Phys. 26, Suppl. 26-3, p. 337.

[37] Berker, A. N. and Nelson, D. R., Phys. Rev. B 19 (1979) 2488.

[38] Mon, K. K. and SaAm, W. F., Phys. Rev. B 23 (1981) 5824.

[39] Desideri, J. P., Laheurte, J. P. and Williams, G. A., Two-phase sound in stratified ${ }^{3} \mathrm{He}-{ }^{4} \mathrm{He}$ mixtures, J. Low. Temp. Phys. 60 (1985).

[40] LAMB, H., Hydrodynamics, sixth edition (Dover publication) p. 370 . 